\title{
Effect of Hemodiafiltration on Self-Reported Sleep Duration: Results from a Randomized Controlled Trial
}

\author{
Maggie Han ${ }^{a}$ b Murilo Guedes ${ }^{a}$ John Larkin ${ }^{a, c}$ Jochen G. Raimann ${ }^{b}$ \\ Ana Beatriz Lesqueves Barra ${ }^{d}$ Maria Eugênia Fernandes Canziani ${ }^{\mathrm{e}}$ \\ Américo Lourenço Cuvello Neto ${ }^{f}$ Carlos Eduardo Poli-de-Figueiredo ${ }^{g}$ \\ Peter Kotanko $^{b, h}$ Roberto Pecoits-Filho ${ }^{a}$
}

a School of Medicine, Pontifícia Universidade Católica do Paraná, Curitiba, Brazil; ${ }^{b}$ Renal Research Institute, Research Department, New York, NY, USA; ' Fresenius Medical Care AG \& Co. KGaA, Bad Homburg, Germany; ${ }^{\mathrm{d} M e d i c a l}$ Office, Fresenius Medical Care, São Paulo, Brazil; ${ }^{e}$ Escola Paulista de Medicina, Universidade Federal de São Paulo, São Paulo, Brazil; f Department of Nephrology, Hospital Alemão Oswaldo Cruz, São Paulo, Brazil; ${ }^{9}$ School of Medicine,

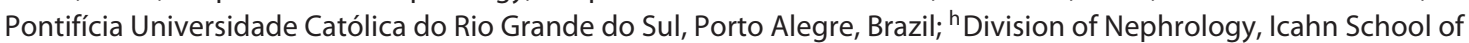
Medicine at Mount Sinai, New York, NY, USA

\section{Keywords}

End-stage kidney disease - End-stage renal disease . Hemodiafiltration · Hemodialysis · Self-reported sleep duration

\begin{abstract}
Introduction: Dialysis patients suffer from poor sleep duration and quality. We examined the self-reported sleep duration in patients randomized to either high-volume hemodiafiltration (HDF) or high flux hemodialysis (HD). Methods: Patients from 13 Brazilian dialysis clinics were enrolled in the HDFIT randomized controlled trial (RCT) investigating the impact of HDF on physical activity and self-reported outcomes. Self-reported sleep duration was taken from patient diaries recording sleep start and end time over a week during baseline, months 3 and 6, respectively. Sleep duration was analyzed by shift and nights relative to dialysis. Results: The HDFIT study enrolled 197 patients; sleep data were available in 173 patients ( 87 HD; 86 HDF). Patients' age was $53 \pm 15$ years, $57 \%$ were white, $72 \%$ were male, $34 \%$ had diabetes, Kt/V was
\end{abstract}

(c) 2019 S. Karger AG, Basel
$1.54 \pm 0.40$, and albumin $3.97 \pm 0.36 \mathrm{~g} / \mathrm{dL}$. Most patients reported sleeping 510-530 min/night. At 3 months, HDF patients slept $513 \pm 71 \mathrm{~min} / \mathrm{night}$, HD patients $518 \pm 76 \mathrm{~min} /$ night. At 6 months, HDF patients slept $532 \pm 74 \mathrm{~min} /$ night, HD patients $519 \pm 80 \mathrm{~min} /$ night. At baseline, 1st shift patients slept $406 \pm 86 \mathrm{~min}$ the night before HD, $534 \pm 64 \mathrm{~min}$ the night after HD, and $496 \pm 99$ min the night between 2 non-HD days. Compared to patients in the 2 nd and 3 rd shifts, patients dialyzed in the $1 \mathrm{st}$ shift slept less in the night before dialysis. Similar patterns were seen after 3 and 6 months. Conclusion: In our RCT, the dialysis modality (HDF vs. HD) had no effect on self-reported sleep duration. In both groups, dialysis in the 1st shift adversely affected self reported sleep duration.

(c) 2019 S. Karger AG, Basel

\section{Introduction}

End-stage kidney disease (ESKD) patients experience a greater burden of disease from sleep disorders compared to the general population [1-3] and chronic kidney

\section{KARGER}

E-Mail karger@karger.com

www.karger.com/bpu
Maggie Han

Renal Research Institute

Research Department, 315 East 62nd Street

4th Floor, New York, NY 10065 (USA)

E-Mail maggie.han@ rriny.com 
disease (CKD) population who do not require renal replacement therapy [4-6]. One of the reasons as to why ESKD patients experience higher rates sleep/wake disorders could be attributed to the dialysis treatment itself [7] . Sleep disorders in CKD and ESKD patients include sleep apnea, both central and obstructive sleep apnea (CSA and OSA), restless leg syndrome, periodic limb movement disorder, and insomnia [8-12]. Poor sleep and certain sleep disorders such as sleep apnea, have also been linked in the decline of renal function and progression of CKD in both the healthy and CKD populations [13-16].

Outcomes that are adversely associated with sleep disorders include quality of life, physical functioning, depression, renal function, and survival $[17,18]$. It has been shown that dialysis patients who have better sleep experience improved quality of life and outcomes compared to those who sleep poorly $[4,19,20]$. Sabbagh et al. [21] found that patients who reported poor sleep exhibited poor physical function after controlling for inflammation and dialysis adequacy.

Factors associated with poor and disordered sleep include fluid overload, uremia, anemia, inflammation, and etiology of renal disease $[22,23]$. In a study by Tada et al. [24], it was found that the dominant type of sleep apnea in hemodialysis (HD) patients is OSA, and that circulating blood urea nitrogen, creatinine, and serum bicarbonate are predictors of OSA. Another study of sleep quality in HD patients found that poor sleepers had higher serum phosphate, PTH, and lower hemoglobin levels compared to good sleepers [10]. Chiu et al. [25] found that worsened sleep is positively correlated with C-reactive protein and Interleukin-1B levels, markers of inflammation, in a study of HD patients.

Given the implications of poor sleep in the ESKD population, interventions to improve sleep have been studied. Unruh et al. [26] reported that neither frequent in-center HD nor nocturnal dialysis demonstrated significant improvements in self-reported sleep. Losso et al. [27] found that fluid overload contributes to the pathogenesis of OSA and CSA and that ultrafiltration attenuates sleep apnea. When looking into different dialysis modalities, it was found that patients on HD had a lower proportion of OSA than those on CAPD and APD. Contrarily, Koch et al. [28] found that conventional day time dialysis patients had the worst sleep compared to nocturnal HD and APD patients.

High volume hemodiafiltration (HDF) is a kidney replacement therapy that is potentially associated with an improvement in fluid status and solute clearance, among other advantages compared to high-flux HD, which could potentially improve disturbances in sleep $[29,30]$. A cross-sectional study found that men receiving HDF had less severe insomnia symptoms compared to those receiving HD [31]. Concrete conclusions were not reached regarding the effect of HDF due to the observational nature of the study. We explored the effect of HDF on sleep using data from a multi-center randomized controlled trial (RCT) comparing HDF to high flux HD. We hypothesized that patients treated with HDF would exhibit longer self-reported sleep duration compared to those treated with HD. Additionally, we attempted to elucidate factors associated with self-reported sleep duration.

\section{Materials and Methods}

\section{Setting and Study Design}

Data from the Brazilian impact of HDF on physical activity and self-reported outcomes (HDFIT) RCT (ClinicalTrials.gov NCT02787161) were used in this post hoc analysis. The detailed RCT methodology was published by Pecoits-Filho et al. [32]. In brief, HDFIT was a prospective, multicenter, unblinded RCT investigating the impact of dialysis modality (HDF vs. HD) on several outcomes including objectively measured physical activity and quality of life. Clinically stable HD patients were recruited from outpatient dialysis centers in the south and southeast regions of Brazil. Patients were required to have been on $\mathrm{HD}$ for 3-24 months prior to randomization. After signing informed consent, patients enrolled in a run-in period of up to 4 weeks during which they received high-flux HD and had baseline assessments for demographic, clinical, social, and selfreported sleep duration parameters. Patients who successfully completed the run-in period were randomized in a 1:1 ratio, stratified by study site and dialysis shift, to either high volume online HDF or continued high-flux HD for a 6-month period. Baseline laboratory samples were taken at the randomization visit. Two study visits were scheduled 3 and 6 months after randomization, where similar assessments as those done during the baseline period were performed.

\section{Ethical Approval}

Prior to study commencement, the protocol, informed consent form, and study documents were approved by Pontifícia Universidade Católica do Paraná ethics review board (central application \# 54926916.7.1001.0020; approval number 1.538.784). The trial was performed in accordance with the Declaration of Helsinki.

\section{Self-Reported Sleep Duration}

As a part of the study, patients were asked to record the time they went to bed and the time they got out of bed for each respective night during the week of data collection; these data were used to compute self-reported sleep duration. Daytime sleep, for example, napping, was not recorded.

\section{Dialysis Shift}

Participating dialysis clinics performed HD/HDF treatments in 3 shifts, the 1st approximately from 6 to 10 a.m., the 2 nd from 10 a.m. to 2 p.m., and the 3rd after 2 p.m. Dialysis start and end times were reported by patients via diary; the distribution of dialysis start times was verified before analysis of dialysis shift. 
Table 1. Descriptive statistics of HDFIT cohort with sleep data at baseline

\begin{tabular}{|c|c|c|c|c|}
\hline & $\begin{array}{l}\mathrm{HD} \\
(n=87)\end{array}$ & $\begin{array}{l}\mathrm{HDF} \\
(n=86)\end{array}$ & $\begin{array}{l}\text { Total } \\
(n=173)\end{array}$ & $p$ value \\
\hline Shift, $n(\%)$ & & & & 0.869 \\
\hline First & $30(34)$ & $28(33)$ & $58(33)$ & \\
\hline Second & $31(36)$ & $34(39)$ & $65(38)$ & \\
\hline Third & $26(30)$ & $24(28)$ & $50(29)$ & \\
\hline Age, years, mean (SD) & $53(14)$ & $52(16)$ & $53(15)$ & 0.619 \\
\hline Gender, $n(\%)$ & & & & 0.426 \\
\hline Male & $60(69)$ & $64(74)$ & $124(72)$ & \\
\hline Race, $n(\%)$ & & & & 0.141 \\
\hline Other & $42(48)$ & $32(37)$ & $74(43)$ & \\
\hline White & $45(52)$ & $54(63)$ & $99(57)$ & \\
\hline Family income, minimum wage, $n(\%)$ & & & & 0.436 \\
\hline$<2 \times$ & $20(23)$ & $13(15)$ & $33(19)$ & \\
\hline $2-4 \times$ & $34(39)$ & $44(51)$ & $78(45)$ & \\
\hline $4-10 \times$ & $26(30)$ & $25(29)$ & $51(29)$ & \\
\hline $10-20 \times$ & $6(7)$ & $3(3)$ & $9(5)$ & \\
\hline$>20 \times$ & $1(1)$ & $1(1)$ & $2(1)$ & \\
\hline Higher education, $n(\%)$ & & & & 0.730 \\
\hline Some college or higher & $18(21)$ & $16(19)$ & $34(20)$ & \\
\hline Distance from the clinic, $\mathrm{km}, n(\%)$ & & & & 0.610 \\
\hline$>25$ & $22(26)$ & $19(22)$ & $41(24)$ & \\
\hline Between 13 and 25 & $19(22)$ & $24(28)$ & $43(25)$ & \\
\hline Between 7 and 13 & $20(23)$ & $23(27)$ & $43(25)$ & \\
\hline$<7$ & $25(29)$ & $19(22)$ & $44(26)$ & \\
\hline Hemoglobin, g/dL, mean (SD) & $11.05(1.71)$ & $11.24(1.63)$ & $11.14(1.67)$ & 0.467 \\
\hline Albumin, g/dL, mean (SD) & $3.98(0.37)$ & $3.96(0.34)$ & $3.97(0.36)$ & 0.746 \\
\hline Creatinine, mg/dL, mean (SD) & $9.28(3.21)$ & $9.39(3.00)$ & $9.34(3.10)$ & 0.824 \\
\hline $\mathrm{Kt} / \mathrm{V}$, mean $(\mathrm{SD})$ & $1.49(0.41)$ & $1.59(0.39)$ & $1.54(0.40)$ & 0.104 \\
\hline $\mathrm{BMI}, \mathrm{kg} / \mathrm{m}^{2}$, mean $(\mathrm{SD})$ & $27.5(5.2)$ & $25.8(4.3)$ & $26.6(4.9)$ & 0.026 \\
\hline Diabetes, $n(\%)$ & $34(39.1)$ & $25(29.1)$ & $59(34.1)$ & 0.165 \\
\hline
\end{tabular}

HD, hemodialysis; HDF, hemodiafiltration; BMI, body mass index.

\section{Categorization of Nights Relative to Dialysis Treatment}

We categorized the nights during the study period as the night before $\mathrm{HD} / \mathrm{HDF}$, after $\mathrm{HD} / \mathrm{HDF}$, and between 2 non-HD days (the latter is commonly known as "long interdialytic interval").

\section{Statistical Analysis}

Data were assessed for normality. Continuous variables are summarized as means and SD or medians and interquartile ranges. Categorical variables are reported as counts and proportions. When appropriate, Student $t$ tests or Mann-Whitney rank-sum U tests were used. Linear mixed effect models were constructed for the analysis of the treatment effect of HDF vs. HD on mean selfreported sleep time duration, allowing random intercept and slopes. Subgroup analyses based on nights in reference to dialysis day and shifts were carried out. For analysis of the determinants of self-reported sleep time duration at baseline, linear mixed effect models were built to account for repeated measurements. For this model, the predictors were explored based on a set of variables determined a priori, which included diabetes as comorbidity, mode of transportation to dialysis clinic categorized as the use of public transportation, shift and nights in reference to dialysis day. Interaction terms between shifts and day-type were allowed in order to address the hypothesis of effect modification of night type effect by shift. All analyses were performed in R software version 3.5.1.

\section{Results}

\section{Patient Characteristics}

The HDFIT study enrolled 197 patients, and sleep data were available in 173 patients ( $87 \mathrm{HD} ; 86 \mathrm{HDF}$ ). Patients' age was $53 \pm 15$ years, $34 \%$ had diabetes, $72 \%$ were males, $57 \%$ were whites, $20 \%$ had higher education (some college or greater), $24 \%$ lived $>25 \mathrm{~km}$ from the clinic, and $19 \%$ had an annual family income of less than twice the minimum wage (Table 1 ). 
Table 2. Self-reported sleep duration (mean \pm SD; in minutes) per dialysis modality and shift

\begin{tabular}{|c|c|c|c|}
\hline & Baseline & 3-month & 6-month \\
\hline \multicolumn{4}{|l|}{ HD vs. HDF } \\
\hline All, $n$ & 173 & 171 & 163 \\
\hline Duration & $512 \pm 71$ & $515 \pm 73$ & $525 \pm 77$ \\
\hline $\mathrm{HD}, n$ & 87 & 83 & 79 \\
\hline Duration & $514 \pm 69$ & $518 \pm 76$ & $519 \pm 80$ \\
\hline $\mathrm{HDF}, n$ & 86 & 88 & 84 \\
\hline Duration & $511 \pm 73$ & $513 \pm 71$ & $532 \pm 74$ \\
\hline$\Delta(\mathrm{HD}-\mathrm{HDF})$ & 3 & 5 & -13 \\
\hline \multicolumn{4}{|l|}{ Dialysis shift } \\
\hline Shift $1, n$ & 58 & 60 & 55 \\
\hline Duration & $468 \pm 52$ & $475 \pm 70$ & $481 \pm 66$ \\
\hline Shift $2, n$ & 65 & 63 & 60 \\
\hline Duration & $531 \pm 74$ & $540 \pm 64$ & $546 \pm 75$ \\
\hline$\Delta($ shift $1-\operatorname{shift} 2)$ & $-62^{*}$ & $-64^{*}$ & $-65^{*}$ \\
\hline Shift $3, n$ & 50 & 48 & 48 \\
\hline Duration & $539 \pm 63$ & $534 \pm 69$ & $550 \pm 71$ \\
\hline$\Delta($ shift $1-\operatorname{shift} 3)$ & $-70^{*}$ & $-58^{*}$ & $-69 *$ \\
\hline$\Delta($ shift $2-$ shift 3$)$ & -8 & 6 & -4 \\
\hline
\end{tabular}

\section{Shift and Self-Reported Sleep Duration}

On average, patients on the 1st shift reported sleeping significantly less than those on shifts 2 or 3 (Table 2 ). This significant shorter self-reported sleep duration is most pronounced during the night before $\mathrm{HD} / \mathrm{HDF}$ (Fig. 1). Patients on the 1st shift also reported more variability in sleep duration with ranges from 400 to 600 min during the nights free of HD/HDF. The patterns of average self-reported sleep duration on the different shifts and nights in relation to dialysis during the baseline period is presented in Figure 2. Patterns observed at 3 and 6 months were very similar (data not shown).

At baseline a linear mixed model, which considered the interaction between shift and nights, was constructed to delineate the effect of shift on the effect of night type; the night before HD for the 1st shift patients was used as reference. During nights between 2 non-HD days, 1st shift patients reported sleeping on average 88 min more than on nights before $\mathrm{HD}$, and during the night after $\mathrm{HD}$ 1 st shift patients reported sleeping on average $122 \mathrm{~min}$ more than on nights before HD. During the night free of $\mathrm{HD}$, shift 2 patients reported sleeping on average $41 \mathrm{~min}$ longer than on nights before $\mathrm{HD}$, and on the night after $\mathrm{HD}$, they reported sleeping on average 29 min longer than the night before HD. Shift 3 patients reported sleeping 12 min less on the night between 2 non-HD days and 10 min less on the night after HD compared to the night before HD. The $p$ value based on the likelihood ratio test in nested models for the interaction between day-type and shift was $<0.0001$.

\section{Association between Self-Reported Sleep Time and $H D F$}

On average, most patients reported sleeping 510-530 min per night. Self-reported sleep duration did not differ significantly between the HD and HDF groups during baseline, 3-month or 6-month follow-up (Table 2). In the HDF group, there was a slight, non-significant increase in self-reported sleep duration at 6 months (Fig. 3).

\section{Effect of HDF treatment on Self-Reported Sleep \\ Duration}

On average, patients reported sleeping the most on the nights after $\mathrm{HD} / \mathrm{HDF}$ and the least on the nights before the HD/HDF treatment (Fig. 4). After examining the effect sizes of HD vs. HDF during all nights, nights after $\mathrm{HD} / \mathrm{HDF}$, nights before $\mathrm{HD} / \mathrm{HDF}$, and nights free $\mathrm{HD} /$ $\mathrm{HDF}$, we found that there was no significant effect of the $\mathrm{HD} / \mathrm{HDF}$ treatment on the average self-reported sleep duration (Fig. 5).

\section{Discussion/Conclusion}

The HDFIT RCT afforded the opportunity to research the effects of dialytic modality on self-reported sleep duration. Our results revealed several important findings. First, dialysis modality did not affect self-reported sleep duration. Second, patients in the 1st shift slept significantly less during the night before HD/HDF compared to 2nd and 3rd shift patients. Third, patients in the 1st shift experienced the greatest variability in self-reported sleep duration on nights before $\mathrm{HD}$ versus nights after $\mathrm{HD}$ and free of HD.

It is interesting to note that the self-reported sleep duration in our patients was $>100$ min higher than previously reports in ESKD patients (range from 340 to $370 \mathrm{~min} / \mathrm{night}$ ) that were determined using other instruments such polysomnography and wrist-based activity trackers $[5,19,33-35]$. This observation raises the question whether self-reported sleep time may systematically over-estimate sleeping time. However, the patients in our study were trained to document the time when they went to bed and when they got up. The time between going to 


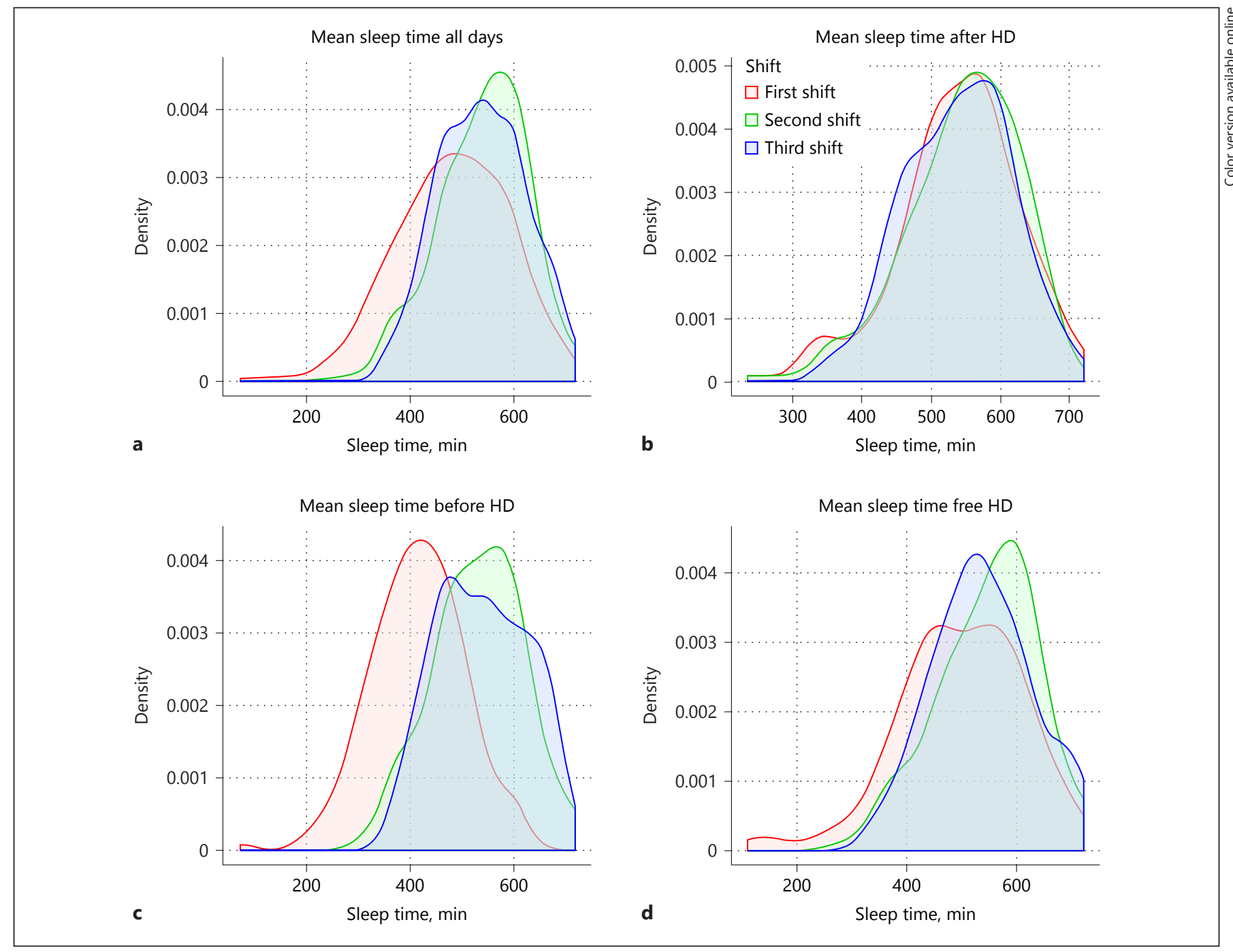

Fig. 1. Density plot of mean self-reported sleep duration (in minutes) across shifts 1-3 of (a) all days, (b) night after HD, (c) night before $\mathrm{HD}$, and (d) night free HD. First shift = 6-10 a.m.; second shift = 10 a.m. to 2 p.m.; Third shift: after 2 p.m. Night free HD is the night between 2 non-HD days. HD, hemodialysis.

bed and falling asleep, the so-called sleep latency time, has been reported to be on average $50 \mathrm{~min}$ in HD patients [10]. In addition to the instruments used to measure sleeping time, population, geographical, and cultural differences may play a role too. While sleep duration, selfreported or objectively measured, has not been previously reported in Brazilian ESKD population, it has been reported in a study of 1,621 HD patients of the Brazilian Unified Health System that over a fifth of Brazilian HD patients surveyed had trouble falling asleep most of the time [36]. We have no reasons to believe that dialysis modality may have impacted the patients' documentation accuracy or precision.
The effect of shift on sleep duration and quality has been studied previously, although the conclusions are not consistent across studies. A small cross-sectional study in Pakistani HD patients found that shift did not have a major effect on sleep scores measured by Pittsburgh Sleep Quality Index [2]. A larger study in $100 \mathrm{HD}$ patients came to the same conclusion that poor sleep (Pittsburgh Sleep Quality Index $>6$ ), restless leg syndrome, and EDS were not associated with dialysis shift [8]. When measuring sleep quantity and quality with actigraphy, Barmar et al. [37] found that being on an early morning dialysis shift was associated with a shorter total sleep duration and greater nightly total sleep time varia- 
Fig. 2. Self-reported sleep duration (in minutes) during different nights presented by shift at the baseline period. Similar patterns were observed at the 3 and 6-month periods. HD, hemodialysis.

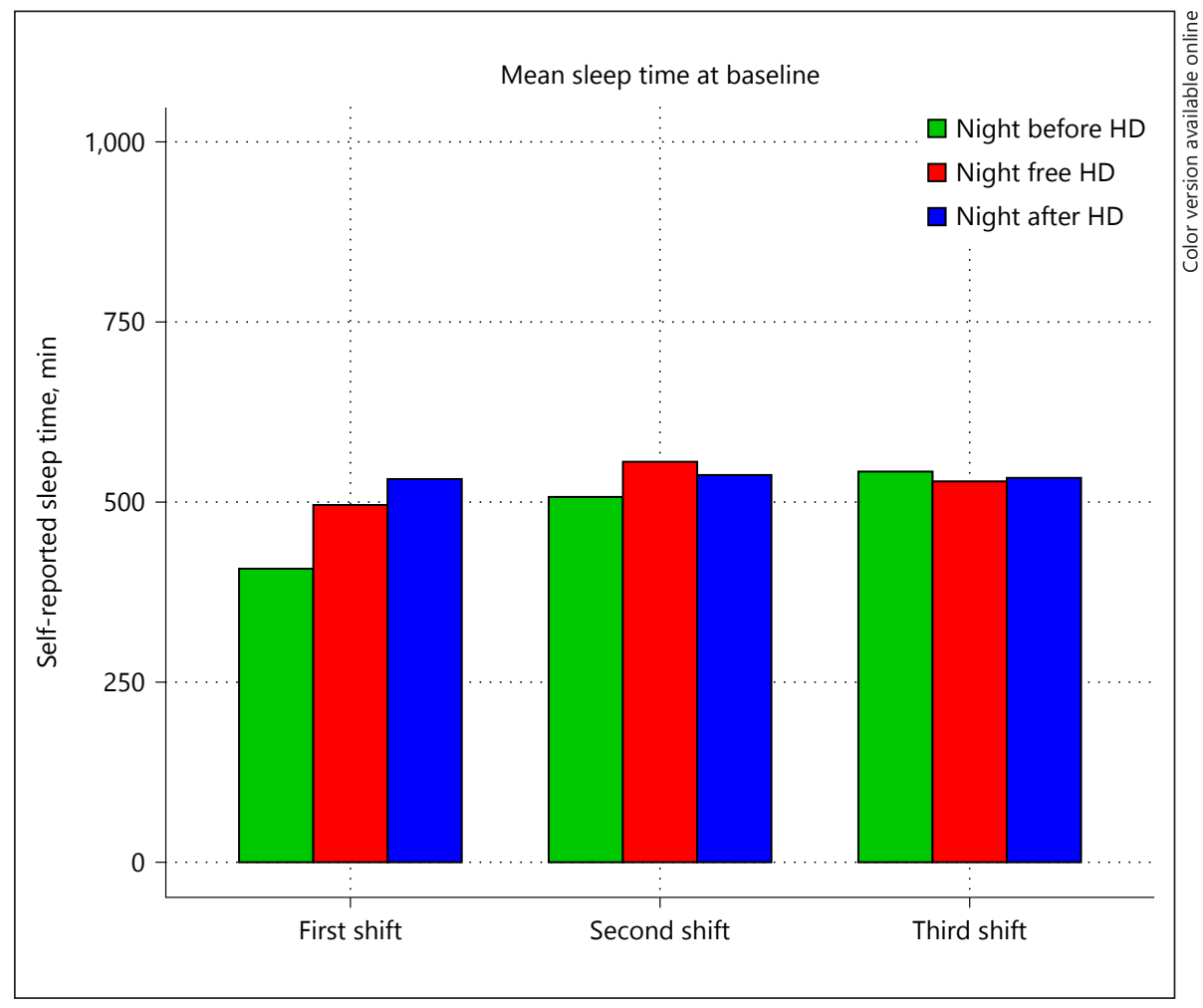

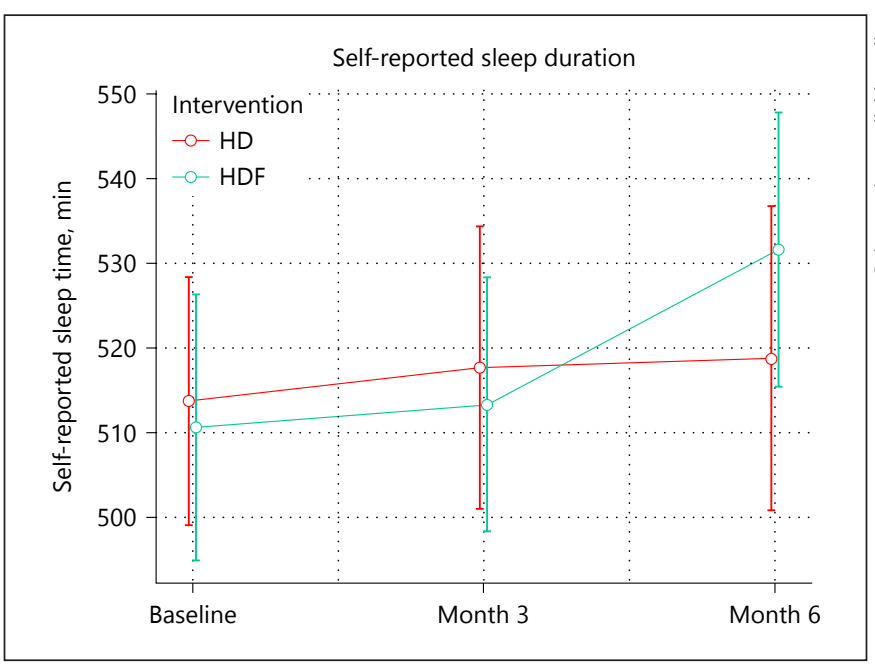

Fig. 3. Self-reported sleep duration (in minutes) of HD and HDF groups at the baseline, 3-month, and 6-month. HD, hemodialysis; $\mathrm{HDF}$, hemodiafiltration.

tion. A larger study in $200 \mathrm{HD}$ patients found that sleep questionnaire scores were associated with receiving dialysis during the last shift of the day [10]. While there are differing results across these studies, it is observed that when using objective measures of sleep such as actigra- phy, the first shift patients are seen to experience the worse overall levels of sleep and the greatest disturbance to the sleep/wake cycles [33, 34, 37]. We observed similar findings in our patients where the patients in the 1st shift were driving the differences in self-reported sleep duration across the different nights of the week (Fig. 2). The effect of shift on sleep duration is most likely due to operational effects, for example, having to wake up earlier to go to an early-morning dialysis session. Work done by Bastos et al. [8] in a Brazilian population showed that there was no significant differences in measures of sleep quality in respect to shift.

More is not always necessarily better when it comes to sleep. Studies have shown that it is detrimental to both quality of life and survival if a patient sleeps less or more than the optimal amount of sleep, which is anywhere from 7 to $8 \mathrm{~h}[38,39]$. In fact, a study done in 3 communities in China found that sleep duration $<6 \mathrm{~h} /$ day and $>10 \mathrm{~h} /$ day tended to be associated with an increased risk of renal hyperfiltration in middle-aged general population. Another study by Yamamoto et al. [40] found that shorter $(\leq 5 \mathrm{~h})$ and longer $(>8 \mathrm{~h})$ sleep duration and poor sleep quality were associated with the incidence of ESKD in CKD patients. The average amount of sleep observed in our population is greater than the 
Fig. 4. Self-reported sleep duration (in minutes) of night before $\mathrm{HD} / \mathrm{HDF}$, night after $\mathrm{HD} / \mathrm{HDF}$, and night free HD/HDF (night between 2 non-treatment days) at the baseline, 3-month, and 6-month. The dashed lines denote HDF treatment group and solid lines denote HD treatment group. Night free HD is the night between 2 nonHD days. HD, hemodialysis; HDF, hemodiafiltration.

Fig. 5. Forest plots of effect sizes of HDF vs. HD. Four categories are shown: overall (all nights), night after dialysis treatment, night before dialysis treatment, and night between 2 non-treatment days. Circle and square denote the effect size at 3 and 6 months respectively; a diamond denotes the total treatment effect. HD, hemodialysis; HDF, hemodiafiltration.
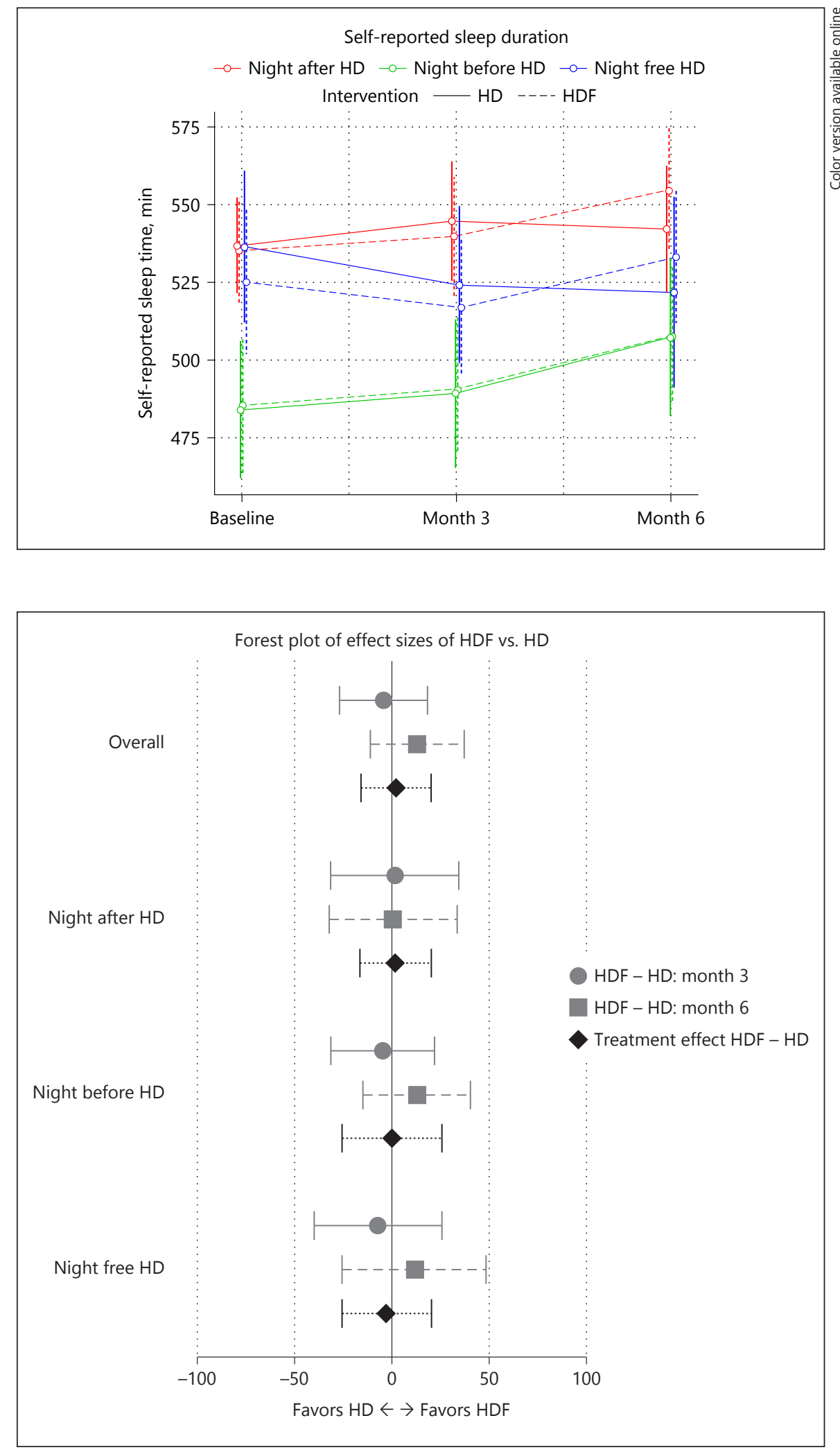
recommended amount for older adults, which is anywhere from 7 to $8 \mathrm{~h}$ per evening.

To the best of our knowledge, this is the first study of the effect of HDF on self-reported sleep duration in an RCT. It has been previously speculated that HDF would have an ameliorating effect on the negative symptoms, such as insomnia, associated with ESKD. Although we did not see a difference across the 2 groups, we did see a non-significant increase in selfreported sleep time in the HDF group at the 6-month follow-up compared to the baseline and 3-month periods. Importantly, based on the concept that HDF provides a very different approach to solute removal with the introduction of the convection component, the lack of differences in self-reported sleep duration between dialysis modalities adds value to the safety profile of HDF.

This study has many strengths, most prominently being a multicenter RCT representative of in-center dialysis patients in the first 2 years of renal replacement therapy who are adherent to treatment. However, there are a few limitations. First, the measure of sleep is not an objective measure, but instead a subjective one. Second, we only measured the "main" sleep phase, the daily nocturnal sleep. We did not collect information on any naps the patient may have taken either during the dialysis treatment or after they returned home from their treatment. Last, we only captured details of self-reported sleep duration, but did not capture parameters of sleep quality.

In conclusion, we describe for the first time equivalent self-reported sleep duration in patients treated with high-flux HD and HDF. Patients in the 1st shift reported sleeping significantly less the pre-dialysis night compared to those in later shifts. Furthermore, 1st shift patients also experienced the greatest differences in selfreported sleep duration between the nights before HD versus after HD and between 2 non-HD days. Operational factors, particularly the time of dialysis initiation, rather than demographic, clinical or biochemical characteristics have a major impact on self-reported sleep duration. Investigation of how to best mitigate these operational limitations on sleep duration should be further investigated to improve sleep in ESKD patients requiring in-center dialysis.

\section{Statement of Ethics}

Please see "Ethical Approvals".

\section{Disclosure Statement}

R.P.-F. is employed by Pontifícia Universidade Católica do Paraná. R.P.-F. is employed by Arbor Research Collaborative for Health, and receives research grants, consulting fees, and honoraria from Astra Zeneca, Novo Nordisc, Akebia, and Fresenius Medical Care. R.P.-F., C.E.P.-F., and M.E.F.C. are recipients of scholarships from the Brazilian Council for Research (CNPq). J.L., M.G., and M.H. are students at Pontifícia Universidade Católica do Paraná. J.L. is an employee of Fresenius Medical Care AG and Co., KGaA, and M.H., P.K., and J.G.R. are employees of Renal Research Institute, a wholly owned subsidiary of Fresenius Medical Care North America. C.E.P.-F. and A.L.C.N. received consulting fees and speaker honorarium from Fresenius Medical Care. C.E.P.-F. received lecture fees and travel support from Fresenius Medical Care, Alexion, Baxter, and Astra Zeneca, and is employed by Pontifícia Universidade Católica do Rio Grande do Sul. A.B.L.B. is an employee of Fresenius Medical Care Brazil. P.K. has share options/ownership in Fresenius Medical Care, received author honorarium from Up-To-Date, and is on the Editorial Board of Blood Purification and Kidney and Blood Pressure Research. M.E.F.C. is an employee by Federal University of São Paulo, and received research grants, consulting fees, and honoraria from Baxter Healthcare and Fresenius Medical Care.

\section{Funding Sources}

This trial was a multicenter investigator-initiated study, whereby the site investigators and principal investigator were not being monetary funded for the conduct of study activities. This project was supported by: (1) the study investigators, (2) the proponent institution Pontifícia Universidade Católica do Paraná, (3) the outpatient dialysis centers, and (4) Fresenius Medical Care. The steering committee was comprised of nephrologists representing site institutions and supporting affiliates.

Investigators were involved in the design of the protocol and performed medical oversight and the coordination of data collection during the trial. The principal investigator provided medical oversight of the conduct of the trial at all sites under the guidance of the steering committee and coordinated the trial management.

The proponent institution Pontifícia Universidade Católica do Parana supported the trial with infrastructure for study management through use of the university's ACRO, hosting of the REDCap eCRF on the university's server, and use of the university's central ERB and Research Council.

The outpatient dialysis centers permitted clinical research at the clinics and supported the trial with their clinical staff who performed data collection and conducted study procedures under the oversight of the site investigators and local trial leadership.

Fresenius Medical Care provided the sites with the infrastructure for the conduct of the trial including HDF machines, dialysis supplies for study participants, BCM machines in clinics without them. Also, they provided some staff for site monitoring. Fresenius Medical Care provided a monetary award to PUCPR's academic clinical research organization (EPICENTER) that performed the 
central management, data acquisition, and monitoring. Fresenius Medical Care and the subsidiary company Renal Research Institute provided support from statistical experts to assist in the analysis of trial data under the oversight of the steering committee. Fresenius Medical Care has supported 3 investigator meetings, as well as 3 steering committee meetings. The leadership of Fresenius Medical Care reviewed and approved the protocol prior to commencement.

\section{Author Contributions}

M.H., R.P.-F., P.K., J.L., J.G.R., and M.G. were responsible for analysis design and hypothesis generation. Data cleaning was performed by M.H. and J.L. Data analysis was performed by M.G., A.B.L.B., A.L.C.N., C.E.P.-F., and R.P.-F. were responsible for the conception/execution of HDFIT. All authors participated in manuscript generation and revision.

\section{References}

1 Agarwal R, Light RP. Sleep and activity in chronic kidney disease: a longitudinal study. Clin J Am Soc Nephrol. 2011 Jun;6(6):125865.

2 Anwar N, Mahmud SN. Quality of sleep in ckd patients on chronic hemodialysis and the effect of dialysis shift. J Coll Physicians Surg Pak. 2018 Aug;28(8):636-9.

3 Parker KP. Sleep disturbances in dialysis patients. Sleep Med Rev. 2003 Apr;7(2):131-43.

4 Roumelioti ME, Argyropoulos C, Buysse DJ, Nayar H, Weisbord SD, Unruh ML. Sleep quality, mood, alertness and their variability in CKD and ESRD. Nephron Clin Pract. 2010; 114(4):c277-87.

5 Ezzat H, Mohab A. Prevalence of sleep disorders among ESRD patients. Ren Fail. 2015 Jul; 37(6):1013-9.

6 Roumelioti ME, Buysse DJ, Sanders MH, Strollo P, Newman AB, Unruh ML. Sleep-disordered breathing and excessive daytime sleepiness in chronic kidney disease and hemodialysis. Clin J Am Soc Nephrol. 2011 May;6(5):986-94.

7 Koch BC, Nagtegaal JE, Kerkhof GA, ter Wee PM. Circadian sleep-wake rhythm disturbances in end-stage renal disease. Nat Rev Nephrol. 2009 Jul;5(7):407-16.

8 Bastos JP, Sousa RB, Nepomuceno LA, Gutierrez-Adrianzen OA, Bruin PF, Araújo ML, et al. Sleep disturbances in patients on maintenance hemodialysis: role of dialysis shift. Rev Assoc Med Bras (1992). 2007 Nov-Dec;53(6): 492-6.

9 Benz RL, Pressman MR, Hovick ET, Peterson DD. A preliminary study of the effects of correction of anemia with recombinant human erythropoietin therapy on sleep, sleep disorders, and daytime sleepiness in hemodialysis patients (The SLEEPO study). Am J Kidney Dis. 1999 Dec;34(6):1089-95.

10 Cengić B, Resić H, Spasovski G, Avdić E, Alajbegović A. Quality of sleep in patients undergoing hemodialysis. Int Urol Nephrol. 2012 Apr;44(2):557-67.

11 Abassi MR, Safavi A, Haghverdi M, Saedi B. Sleep disorders in esrd patients undergoing hemodialysis. Acta Med Iran. 2016 Mar; 54(3):176-84

12 Hsu CY, Lee CT, Lee YJ, Huang TL, Yu CY, Lee LC, et al. Better sleep quality and less daytime symptoms in patients on evening hemo- dialysis: a questionnaire-based study. Artif Organs. 2008 Sep;32(9):711-6.

13 Kim CW, Chang Y, Sung E, Yun KE, Jung HS, Ko BJ, et al. Sleep duration and quality in relation to chronic kidney disease and glomerular hyperfiltration in healthy men and women. PLoS One. 2017 Apr;12(4):e0175298.

14 Lee YC, Hung SY, Wang HK, Lin CW, Wang $\mathrm{HH}$, Chen SW, et al. Sleep apnea and the risk of chronic kidney disease: a nationwide population-based cohort study. Sleep (Basel). 2015 Feb;38(2):213-21.

15 Li J, Huang Z, Hou J, Sawyer AM, Wu Z, Cai J, et al. Sleep and ckd in chinese adults: A cross-sectional study. Clin J Am Soc Nephrol. 2017 Jun;12(6):885-92.

16 Sakaguchi Y, Shoji T, Kawabata H, Niihata K, Suzuki A, Kaneko T, et al. High prevalence of obstructive sleep apnea and its association with renal function among nondialysis chronic kidney disease patients in Japan: a cross-sectional study. Clin J Am Soc Nephrol. 2011 May;6(5):995-1000.

17 Rodriguez L, Tighiouart H, Scott T, Lou K, Giang L, Sorensen E, et al. Association of sleep disturbances with cognitive impairment and depression in maintenance hemodialysis patients. J Nephrol. 2013 Jan-Feb;26(1):101-10.

18 Ricardo AC, Knutson K, Chen J, Appel LJ, Bazzano L, Carmona-Powell E, et al.; Chronic Renal Insufficiency Cohort (CRIC) Study Investigators. The association of sleep duration and quality with ckd progression. J Am Soc Nephrol. 2017 Dec;28(12):3708-15.

19 Parker KP, Kutner NG, Bliwise DL, Bailey JL, Rye DB. Nocturnal sleep, daytime sleepiness, and quality of life in stable patients on hemodialysis. Health Qual Life Outcomes. 2003 Nov;1(1):68.

20 Unruh M, Kurella Tamura M, Larive B, Rastogi A, James S, Schiller B, et al. Impact of sleep quality on cardiovascular outcomes in hemodialysis patients: results from the frequent hemodialysis network study. Am J Nephrol. 2011;33(5):398-406.

21 Sabbagh R, Iqbal S, Vasilevsky M, Barré P. Correlation between physical functioning and sleep disturbances in hemodialysis patients. Hemodial Int. 2008 Oct;12 Suppl 2:S20-4.

22 Edalat-Nejad M, Jafarian N, Yousefichaijan P. Diabetic nephropathy: A strong predictor of sleep quality in hemodialysis patients. Saudi J Kidney Dis Transpl. 2014 Jul;25(4):774-80.

23 Hao G, Lu W, Huang J, Ding W, Wang P, Wang L, et al. Predialysis fluid overload linked with quality of sleep in patients undergoing hemodialysis. Sleep Med. 2018 Nov;51: 140-7.

24 Tada T, Kusano KF, Ogawa A, Iwasaki J, Sakuragi S, Kusano I, et al. The predictors of central and obstructive sleep apnoea in haemodialysis patients. Nephrol Dial Transplant. 2007 Apr;22(4):1190-7.

25 Chiu YL, Chuang YF, Fang KC, Liu SK, Chen HY, Yang JY, et al. Higher systemic inflammation is associated with poorer sleep quality in stable haemodialysis patients. Nephrol Dial Transplant. 2009 Jan;24(1): 247-51.

26 Unruh ML, Larive B, Eggers PW, Garg AX, Gassman JJ, Finkelstein FO, et al.; FHN Trial Group. The effect of frequent hemodialysis on self-reported sleep quality: Frequent Hemodialysis Network Trials. Nephrol Dial Transplant. 2016 Jun;31(6):984-91.

27 Losso RL, Minhoto GR, Riella MC. Sleep disorders in patients with end-stage renal disease undergoing dialysis: comparison between hemodialysis, continuous ambulatory peritoneal dialysis and automated peritoneal dialysis. Int Urol Nephrol. 2015 Feb;47(2): 369-75.

28 Koch BC, Nagtegaal JE, Hagen EC, Wee PM, Kerkhof GA. Different melatonin rhythms and sleep-wake rhythms in patients on peritoneal dialysis, daytime hemodialysis and nocturnal hemodialysis. Sleep Med. 2010 Mar;11(3):242-6.

29 Basile C, Davenport A, Blankestijn PJ. Why choose high volume online post-dilution hemodiafiltration? J Nephrol. 2017 Apr;30(2): 181-6.

30 Canaud B, Koehler K, Bowry S, Stuard S. What is the optimal target convective volume in on-line hemodiafiltration therapy? Contrib Nephrol. 2017;189:9-16.

31 Orasan OH, Saplontai AP, Cozma A, Racasan $S$, Kacso IM, Rusu CC, et al. Insomnia, muscular cramps and pruritus have low intensity in hemodialysis patients with good dialysis efficiency, low inflammation and arteriovenous fistula. Int Urol Nephrol. 2017 Sep;49(9): 1673-9. 
32 Pecoits-Filho R, Larkin JW, Poli-de-Figueiredo CE, Cuvello Neto AL, Barra AB, Canhada $S$, et al.; HDFIT Study Investigators. Design and methodology of the impact of HemoDiaFIlTration on physical activity and self-reported outcomes: a randomized controlled trial (HDFIT trial) in Brazil. BMC Nephrol. 2019 Mar;20(1):98.

33 Han M, Williams S, Mendoza M, Ye X, Zhang H, Calice-Silva V, et al. Quantifying physical activity levels and sleep in hemodialysis patients using a commercially available activity tracker. Blood Purif. 2016;41(1-3): 194-204.

34 Williams S, Han M, Ye X, Zhang H, MeyringWösten A, Bonner M, et al. Physical activity and sleep patterns in hemodialysis patients in a suburban environment. Blood Purif. 2017; 43(1-3):235-43.

35 Abreo AP, Dalrymple LS, Chertow GM, Kaysen GA, Herzog CA, Johansen KL. Predialysis volume overload and patient-reported sleep duration and quality in patients receiving hemodialysis. Hemodial Int. 2017 Jan;21(1):133-41.

36 Moreira TR, Giatti L, Cesar CC, Andrade EI, Acurcio FA, Cherchiglia ML. Health self-assessment by hemodialysis patients in the Brazilian Unified Health System. Rev Saude Publica. 2016;50(0):10.

37 Barmar B, Dang Q, Isquith D, Buysse D, Unruh $\mathrm{M}$. Comparison of sleep/wake behavior in CKD stages 4 to 5 and hemodialysis populations using wrist actigraphy. Am J Kidney Dis. 2009 Apr;53(4):665-72.
38 Sung SA, Hyun YY, Lee KB, Park HC, Chung W, Kim YH, et al.; KNOW-CKD Study Investigators. Sleep duration and health-related quality of life in predialysis ckd. Clin J Am Soc Nephrol. 2018 Jun;13(6):858-65.

39 Liu TZ, Xu C, Rota M, Cai H, Zhang C Shi MJ, et al. Sleep duration and risk of all-cause mortality: A flexible, non-linear, meta-regression of 40 prospective cohort studies. Sleep Med Rev. 2017 Apr;32: 28-36.

40 Yamamoto R, Shinzawa M, Isaka Y, Yamakoshi E, Imai E, Ohashi Y, et al.; CKD-JAC Investigators. Sleep quality and sleep duration with ckd are associated with progression to eskd. Clin J Am Soc Nephrol. 2018 Dec; 13(12):1825-32. 